\title{
Special Issue: 21st International Conference on Multiple Criteria Decision Making; Articles on multiobjective optimization
}

\author{
Kaisa Miettinen · Serpil Sayin
}

Received: 30 January 2013 / Accepted: 31 January 2013 / Published online: 13 February 2013

C Springer Science+Business Media New York 2013

This special issue devoted to multiobjective optimization contains seven carefully reviewed papers selected from the submissions based on presentations given at the 21st International Conference on Multiple Criteria Decision Making (MCDM) which was held in Jyväskylä, Finland, in June 13-17, 2011. Biennial International Conferences on MCDM are the main events of the International Society on Multiple Criteria Decision Making for researchers and practitioners in the field. The conference in Jyväskylä was a particular success with 245 oral and 15 poster presentations organized into 68 sessions besides plenary and award sessions and attended by 311 registered participants. An excellent conference organization accompanied by the spectacular midnight sun led to memorable experiences for participants. The research content of the conference was very rich. Therefore, we believe the articles in this special issue constitute a fine representative sample of recent research achievements at the crossroad of global and multiobjective optimization.

The articles you will find in this issue span a wide spectrum of traditionally difficult research areas in multiobjective optimization, including discrete, stochastic and parametric optimization. Application of stochastic optimization to multiobjective portfolio optimization, incorporation of data envelopment analysis into multiobjective evolutionary algorithms and introduction of innovization (innovation through optimization) are other topics covered in this special issue.

In the article "Higher and Lower-level Knowledge Discovery from Pareto-optimal Sets," Sunith Bandaru and Kalyan Deb build on the concept of innovization, which refers to innovation through optimization by distinguishing between higher and lower level innovization. Their findings are promising for knowledge discovery in challenging engineering design problems.

K. Miettinen $(\varangle)$

Department of Mathematical Information Technology, University of Jyvaskyla,

P.O. Box 35 (Agora), 40014 University of Jyvaskyla, Finland

e-mail: Kaisa.Miettinen@jyu.fi

S. Sayin

College of Administrative Sciences and Economics, Koc University, Sariyer, 34450 Istanbul, Turkey

e-mail: SSAYIN@ku.edu.tr 
In the article "A Stochastic Programming Approach to Multicriteria Portfolio Optimization," Ceren Tuncer Şakar and Murat Köksalan work on the multiobjective multi period portfolio optimization problem using stochastic programming. Expected return, conditional value at risk and liquidity are the three objectives brought together in this formulation. Their computational analysis conducted on data that comes from Istanbul Stock Exchange provides valuable insights for researchers in this field.

In the article "Interactive Procedure for a Multiobjective Stochastic Discrete Dynamic Problem," Maciej Nowak and Tadeusz Trzaskalik propose a dynamic optimization approach to the stochastic multiobjective optimization problem. The procedure uses the Bellman principle to identify the set of Pareto optimal solutions in its first step. The interactive approach is applied to find the most preferred solution in the second step. Applicability of the procedure is verified via a real-world example.

In the article "A Variational Approach to Define Robustness for Parametric Multiobjective Optimization Problems," Katrin Witting, Sina Ober-Blöbaum and Michael Dellnitz use calculus of variations to model Pareto optimal points that are robust to parametric changes in the objective function. This leads to a new definition of robustness in multiobjective optimization.

In the article "Finding all Nondominated Points of Multi-objective Integer Programs," Banu Lokman and Murat Köksalan introduce two algorithms that can obtain the entire Pareto optimal set building on earlier work in this domain by Sylva and Crema published in 2004. The authors demonstrate significant improvements in computational performance on multiobjective knapsack, shortest path and spanning tree instances.

In the article "Utilizing Expected Improvement and Generalized Data Envelopment Analysis in Multi-Objective Genetic Algorithms" Yeboon Yun and Hirotaka Nakayama suggest incorporating generalized data envelopment analysis to assess the fitness of a population member whereas expected improvement is used in offspring selection in a multiobjective genetic algorithm. Comparisons to leading multiobjective evolutionary algorithms including SPEA2 and NSGA-II on several test problems indicate that the approach may be promising.

In the article "Global Search Perspectives for Multiobjective Optimization" Alberto Lovison adopts Stephen Smale's global analysis framework and formulates a multiobjective version of the necessity of generating everywhere dense sample sequences. He also introduces a globally convergent algorithm assuming the Lipschitz constant of the determinant of the Jacobian is known.

The guest editors want to warmly thank authors who showed interest in this special issue and all the reviewers who accepted the invitation to provide their expertise. Thanks to the carefully prepared reviews containing valuable comments and the commitment of the authors whose papers were accepted to be revised, this special issue contains papers that hopefully are useful and interesting to the readers. Finally, thanks to the two Editors in Chief Panos Pardalos and Sergiy Butenko and Rameshbabu Rathinam from Springer for valuable help.

Kaisa Miettinen and Serpil Sayin, Guest Editors 